\title{
DEL MALESTAR AL COMPROMISO: LOS FACTORES INDIVIDUALES EN LA SATISFACCIÓN LABORAL DE LOS DOCENTES*
}

\author{
Manuel Reyes Santana, \\ de la Universidad de Huelva
}

\begin{abstract}
RESUMEN: La preocupación por la satisfacción laboral del profesorado ha ido cambiando desde un enfoque centrado en el malestar hacia una orientación más positiva que focaliza el bienestar como núcleo de interés. En ambas perspectivas son considerados múltiples factores, tanto de índole personal como ambiental. Este artículo recoge una reflexión sobre algunos de los factores individuales que inciden en la satisfacción laboral docente y propone varias sugerencias para su análisis. Se analizan después algunas de las características que deberían reunir posibles modelos teóricos orientados a explicar la satisfacción laboral del profesorado. Por último, se ofrecen algunas orientaciones para una intervención formativa que promueva el bienestar docente.

Palabras claves: Malestar docente. Compromiso docente. Satisfacción laboral.
\end{abstract}

\section{DEL MALESTAR AL BIENESTAR, UNA TRANSICIÓN SUBLIME}

Con denominaciones diferentes, la preocupación por la satisfacción laboral del profesorado es un tema recurrente en el ámbito de la investigación y de la literatura pedagógica. Aparece cada cierto tiempo y cobra auge para sufrir después un declive, quizás en función de ciertas modas o como consecuencia de que otros temas son priorizados por las convocatorias oficiales de investigación. En los últimos años, la investigación ha vuelto a focalizar el problema, como muestran muchos aportes recientes (Jepson y Forrest, 2006; OPECH, 2008; Parra, 2007; Reyes y Cornejo, 2008; UnESCO, 2005;

\footnotetext{
* Artigo recebido em 31/05/2012 e aprovado em 31/05/2012.
} 
entre otros muchos) o el hecho de que la última reunión del Observatorio Internacional de la Profesión Docente (OBIPD), celebrada en Barcelona en abril de 2012, haya centrado la discusión en ese tema y en su situación en distintos países.

Como se ha constatado en la reunión científica del OBIPD, entre las apariciones sucesivas de esa preocupación por la satisfacción o el estado de los docentes, el mismo objeto de estudio ha ido variando. Si cuando emerge por primera se refiere al malestar, poco a poco, la investigación se irá centrando, cada vez más, en el bienestar. En cierto modo, se pasa de una perspectiva de déficit a una perspectiva de desarrollo. Esa transición tiene una gran importancia: si antes eran estudiados los docentes incómodos o insatisfechos con su trabajo, ahora son estudiados los que tienen un grado elevado de satisfacción laboral. Por lo tanto, al variar el objeto también se varían las preguntas de investigación. Ya no interesa tanto cuáles son las fuentes del malestar como conocer qué factores producen bienestar. De ese cambio se deriva otro también importante: de los resultados de investigación sobre malestar se pueden deducir acciones prácticas consistentes que eviten de los factores nocivos; de la investigación sobre bienestar se deducen acciones orientadas a promover los factores saludables. Si se me permite la extrapolación, se ha pasado de estudiar la enfermedad a estudiar la salud. De hecho, el malestar docente, tal como venía siendo considerado, es un estado de ausencia de salud, si consideramos a ésta en su acepción más integral y compleja.

Considerando la insatisfacción y la satisfacción laboral como dos extremos de una misma dimensión, la investigación, durante mucho tiempo, ha desviado su atención hacia el extremo negativo (insatisfacción) y ahora lo hace preferencialmente hacia el extremo positivo (satisfacción). La contraposición de ambos enfoques se hace patente en los conceptos que conforman el campo lexical de cada uno de los dos extremos considerados. Al estudio del primero se asocian términos como burnout, malestar, surmenage, estrés, depresión, ansiedad. Por el contrario, al considerar el extremo positivo aparecen términos como bienestar, satisfacción, compromiso, resiliencia etc.

Evidentemente, el cambio de enfoque debe tener una incidencia notable no sólo en los objetivos de investigación o en la terminología empleada, sino también en los programas formativos. Así, un enfoque centrado en la insatisfacción suscribe una actitud remediativa y debe proveer al profesorado en formación herramientas de afrontamiento (coping). Por el contrario, un enfoque centrado en la satisfacción adopta una actitud 
evolutiva, de crecimiento, y debe proveer al profesorado herramientas para el desarrollo profesional.

En este sentido, se puede considerar que el desarrollo profesional debería ir acompañado de un incremento en la satisfacción laboral o, lo que es lo mismo, del logro progresivo de un estado de bienestar. Esto incorpora una dimensión humanista al concepto de desarrollo profesional que lo contrapone a una conceptuación meramente técnica del mismo. Es el componente personal del desarrollo profesional.

\section{CONTRA EL DETERMINISMO}

En líneas generales, parece cierta la afirmación de que las características del ambiente de trabajo se constituyen en causa suficiente para provocar el malestar o el bienestar de los docentes. El clima laboral, que constituye un concepto amplio en su significado, hace referencia a una amalgama de factores que incide directamente en las relaciones, las formas de comunicación, la satisfacción, la motivación y, en general, la cultura organizativa y profesional: el clima laboral es el medio ambiente humano y físico, es el conjunto de variables, cualidades, atributos o propiedades relativamente permanentes en un ambiente de trabajo concreto (Gonçalves, 1997).

Ese ambiente de trabajo incide en el grado de satisfacción y, a través de ésta, en el comportamiento de las personas que configuran la organización, favoreciendo o inhibiendo el compromiso, la dedicación personal y conductas específicas como el absentismo o la colaboración. Pero, a su vez, el clima es, al menos en parte, resultado de esas conductas y del compromiso y actitudes de las personas que comparten ese ambiente de trabajo. Incluso, cuando son analizadas las condiciones "objetivas" de trabajo, concluimos que se produce esa situación paradójica en el ámbito de la enseñanza. El nuestro es un campo laboral en el que, en gran medida, es el mismo docente quien decide una parte de las condiciones de trabajo. El grado de libertad para construir el ambiente interno del aula es muy elevado, incluso en los sistemas escolares centralizados. Por otro lado, el ambiente externo al aula (las relaciones con los colegas, las condiciones físicas del edificio y sus dependencias etc.) depende, en algunos de sus factores, de las personas que trabajan en él. Así, el clima de trabajo, que tanta incidencia tiene en el grado de satisfacción y bienestar, no es más que el resultado de las relaciones que se establecen entre los colegas y entre éstos y el medio laboral.

Como decía, entre clima y satisfacción laboral se establece una relación circular, mutua. Un clima de colaboración, por ejemplo, sólo 
es posible si los que participan de la acción se muestran favorables a cooperar. A su vez, ese clima, generado por las actitudes colaboracionistas, alimentará conductas individuales de cooperación. Se establece, así, una relación circular entre clima y satisfacción, de forma que uno incide en la otra y viceversa. Eso hace suponer que en situaciones con climas laborales favorables y grados positivos de satisfacción, ambas entidades mejoran progresivamente. Al contrario, con climas laborales desfavorables y estado de malestar en los trabajadores, se produce un empeoramiento progresivo tanto del clima como de ese estado personal negativo, dado que ambos se realimentan. Esta posición es, sin embargo, determinista y no parece la más adecuada para el desarrollo profesional y organizativo porque supone que la evolución de la institución educativa tenderá a la polarización y dependerá enteramente de su estado inicial. Por el contrario, hemos de considerar que, por encima de esa reciprocidad existente entre clima y satisfacción laboral, median otras variables igualmente relevantes (el compromiso, el sentido de responsabilidad etc) que pueden romper la determinación causal. Eso permite que ambientes inapropiados y situaciones de malestar docente sean estados reversibles, transformables, aunque también implica la posibilidad de deterioro de ambientes favorables y el tránsito de un estado de bienestar a su opuesto. En otros términos, la relación circular entre clima de trabajo y satisfacción laboral no es inmediata, sino mediada por factores personales que, potencialmente, pueden revertir la influencia, introduciendo elementos que rompen la relación causal entre ambas entidades. Cuando actúa esa potencialidad de transformar lo negativo hacia estadios evolutivos más satisfactorios, se produce un fenómeno de resiliencia, es decir, de superación de las condiciones desfavorables mediante la incorporación del sentido de desafío. Esos factores personales han originado entonces la ruptura de la relación causal entre un clima laboral desfavorable y un estado de malestar, sea éste individual o compartido.

La resiliencia actúa como elemento de ruptura y como factor terapéutico, en el sentido de detener o prevenir el estado de malestar al que abocaba la relación negativa entre clima y satisfacción.

La experiencia personal de cualquier docente le permite reconocer situaciones de esta índole y quizás también de la opuesta: centros escolares en los que se ha producido un giro histórico positivo hacia estadios de desarrollo institucional y personal más elevados y, en el sentido opuesto, establecimientos docentes que han pasado de ser referentes, en relación con ambientes favorables de trabajo y compromiso de sus componentes, a ser centros mediocres y en declive. Esas transiciones pasan a formar parte de la 
historia institucional y, en cierta medida, pueden condicionar la evolución posterior del establecimiento. La narrativa institucional -historias de vida de los centros elaboradas a partir de los relatos de quienes participan de ellos y de los datos textuales que ofrecen sus documentos organizativos- puede poner de relieve esas transiciones y trazar tendencias y orientaciones.

En definitiva, hay que considerar al ambiente de trabajo como un elemento primordial, pero dinámico, complejo y mudable, que afecta y es afectado por los factores individuales y colectivos del personal adscrito a la institución, los cuales se sitúan en un plano jerárquico superior, de forma que éstos pueden variar las condiciones de trabajo, al menos en la medida precisa, para reorientar el rumbo institucional. No existe, por lo tanto, una determinación causal entre clima y satisfacción laboral en las instituciones escolares, puesto que, en general, los sistemas educativos otorgan a los docentes un grado de libertad suficiente para transformar el clima laboral.

\section{LOS FACTORES PERSONALES Y LA SATISFACCIÓN DOCENTE}

No debe parecer que las anteriores reflexiones se orientan a responsabilizar al profesorado de su propio estado de bienestar o malestar. De hecho, las condiciones de trabajo incluyen ese clima laboral en el que la acción personal y colectiva inciden directamente, pero también incorpora otros factores que escapan a la acción y a las actitudes y voluntades de los docentes: el horario, el salario, el régimen administrativo etc dependen de la normativa emanada de los poderes legislativos y, por delegación de éstos, de la Administración educativa. Esos factores, que no son directamente controlables, conforman el caldo de cultivo de actitudes favorables o desfavorables en el personal, de forma que la disponibilidad o el compromiso pueden verse seriamente afectados cuando esas condiciones son evaluadas negativamente. Será precisamente la evaluación que el docente hace de la situación la que dará origen a un estado más o menos satisfactorio en relación con su desempeño.

Ya Maslach y Jackson (1983) consideraron que, además de los factores contextuales, era esencial la participación de esos factores personales en el desarrollo del burnout. Se referían expresamente a las cualidades de la personalidad y a las habilidades puestas en juego para afrontar las demandas del contexto. Pero, no sólo serán ese tipo de factores, de origen personal, los que incidirán en la satisfacción laboral. Será la percepción de la realidad, la forma como el docente siente su situación, el elemento mediador más relevante en el sentimiento de satisfacción. 
De hecho, la satisfacción laboral se define en la actualidad, de forma genérica, como "la actitud del trabajador frente a su propio trabajo, [que] está basada en las creencias y valores que el trabajador desarrolla de su propio trabajo." (Robbins, 1999: 151) Por tanto, por encima de las condiciones "objetivas" de trabajo, se sitúan la percepción personal que el individuo tiene de las mismas y su voluntad de que exista o no una correlación directa entre esa percepción y su desempeño. Además del dominio de las habilidades necesarias para el ejercicio profesional, esa percepción personal y el nivel de compromiso constituyen, posiblemente, los tres tipos de factores con mayor peso en lo que podríamos denominar "buena práctica docente". Consideradas en conjunto, las relaciones entre esas tres variables explicarían que docentes distintos actuaran de forma desigual ante situaciones similares y se situaran en posiciones diferentes en la dimensión malestar-bienestar.

Por surgir en escenarios industriales o por estar orientados a campos laborales distintos de la enseñanza, los modelos de satisfacción laboral no contemplan normalmente esas relaciones tan complejas. En efecto, en líneas generales, se pueden distinguir tres enfoques sobre satisfacción laboral que ignoran ciertos factores que he sugerido como fundamentales. El primero de esos enfoques supone que la satisfacción es la resultante del balance entre lo esperado del trabajo (las expectativas) y lo que realmente recibe del mismo. Una segunda tendencia concibe la satisfacción como el resultado de la comparación entre lo que la persona aporta al trabajo (el desempeño, la dedicación) y lo que recibe a cambio. La tercera perspectiva considera la incidencia de dos tipos de factores, los de higiene o mantenimiento (remuneración, sistema de supervisión, condiciones materiales, relaciones interpersonales), que son extrínsecos al trabajo y producen efectos negativos sobre la motivación cuando no alcanzan un nivel apropiado, y los motivadores (posibilidades de ascenso, reconocimiento, logros personales, interés por la tarea), que son intrínsecos al trabajo e incrementan la satisfacción y la disponibilidad cuando llegan a un grado apropiado. Como se ve, en los tres tipos de teorías aparece el término recibir: lo que percibe el trabajador se constituye en moneda de cambio, en referente esencial para la satisfacción, con independencia de que lo que se compare sea lo que se espera, lo que se aporta o una mezcla heterogénea de factores. Estas posiciones materialistas no parecen aplicables enteramente a las profesiones y oficios relacionados con lo social, donde la satisfacción debe incorporar otros elementos personales muy asociados a las relaciones interpersonales, a los logros de los otros o a la mejora de las condiciones de vida de los demás. Los otros, los usuarios del servicio (educativo, sanitario, social) no forman parte de aquellos modelos 
que parece necesario desechar o, al menos, modificar sustancialmente para que sean aplicables a nuestros ámbitos laborales.

En todo caso, sea cual sea la perspectiva adoptada, la satisfacción laboral, el estado de bienestar o malestar en el trabajo, es el resultado de la evaluación personal que el trabajador hace de los factores incidentes en el mismo. Siempre situamos, por encima de los términos comparativos, el hecho de que son factores subjetivos los que contribuyen a construir el bienestar o el malestar. Muchas de las investigaciones sobre satisfacción en el trabajo se refieren, por eso, a actitudes, emociones y percepciones. De hecho, el estilo individual con que cada persona evalúa la realidad es esencial para el bienestar docente. Sigue siendo una prueba evidente de esto el hecho de que, en ambientes similares, dos personas pueden tener sentimientos opuestos de satisfacción o insatisfacción. Lo que para unas personas es una amenaza del medio, para otras constituye un reto, un estímulo, un desafío satisfactorio. Con ello, se corrobora que los factores personales se sitúan por encima de los factores ambientales en el desarrollo del bienestar (o de su opuesto, el malestar), es decir, que el componente personal es esencial en el afrontamiento de las situaciones laborales.

Consideremos, a título de ejemplo, algunos de esos factores individuales, aun sabiendo que quedan otros muchos posibles fuera de este análisis.

Una situación rutinaria ocasiona tedio en las personas con más propensión al cambio y con mayor tolerancia de la incertidumbre, mientras que genera seguridad a las personas con tendencias opuestas. Esta cuestión puede constituir, de hecho, un factor limitante en el desarrollo de innovaciones. Tal vez por esa razón hallamos docentes muy innovadores y otros que prefieren la seguridad que les proporciona la rutina. De ahí deducimos que factores o situaciones opuestas (rutina y cambio) pueden ser igualmente generadoras de insatisfacción o de satisfacción laboral. El mismo sentido tiene lo que ha sido llamado "pobreza de rol", entendida como la situación de un trabajador con escasas funciones y una relevancia nula en la organización.

Algo similar a lo que ocasiona la rutina sucede con relación a la mayor o menor disponibilidad de recursos materiales. Ante esa falta de recursos, un docente puede reaccionar de forma negativa, limitando su repertorio didáctico, mientras que a otro la carencia lo puede estimular a la realización de recursos propios, la búsqueda de alternativas, la movilización en definitiva. Se produce, por lo tanto, un inconformismo generador, mientras que en el primero se producía, ante el mismo hecho, una resignación sumisa. 
Esta diferenciación puede ser debido a factores personales como la orientación cognitiva de los docentes, a la que se refirieron Huber y Roth (1990), sugiriendo la existencia de dos estilos u orientaciones en el profesorado:

Docentes orientados a la certidumbre, que prefieren situaciones claras, seguras y ciertas y que orientan su aprendizaje de forma personal, evitando exponer sus ideas a la crítica de otros para poder preservarlas.

Docentes orientados a la incertidumbre, que prefieren comprometerse en situaciones más ambiguas, cuyos resultados no sean tan ciertos ni previstos, orientando el aprendizaje hacia tareas en las que es necesario compartir los puntos de vista y las creencias personales.

También Korthagen (1988) distinguió dos tipos de orientación del aprendizaje en el profesorado:

Docentes con orientación interna, que prefieren aprender por sí mismos y aceptan de mal grado las directrices externas.

Docentes con orientación externa, que prefieren aprender con el asesoramiento o la asistencia de otras personas que faciliten los contenidos, las tareas o las estrategias.

Los dos tipos de Korthagen se identifican en gran medida con los propuestos por Huber y Roth y pueden explicar una mayor o menor propensión a tomar las situaciones escolares como eventuales agentes estresores. Así, un docente con orientación interna normalmente se sentirá incómodo en situaciones de cooperación y trabajo en equipo, que, sin embargo, son preferidas por los que presentan una orientación externa.

\section{HACIA LA CONSTRUCCIÓN DE UN MODELO EXPLICATIVO DEL BIENESTAR DOCENTE}

Como anuncié al inicio, no pretendo ofrecer aquí un modelo acabado de satisfacción docente, sino ofrecer algunas reflexiones previas a la construcción de un modelo y algunas pautas sobre las características esenciales de ese posible modelo.

Evidentemente, cualquier modelo explicativo del bienestar ha de incorporar los factores personales a los que vengo haciendo referencia. Como los analizados en el epígrafe anterior, podríamos considerar otras muchas variables de tipo personal. Por citar algunas de las que deberían ser analizadas en la construcción de un modelo, mencionaré el grado de madurez personal, el estadio de aprendizaje, el estilo en el uso de la experiencia como fuente de aprendizaje o sabiduría, la naturaleza intrínseca o extrínseca de las motivaciones laborales, el pensamiento y las creencias en relación a la enseñanza, el estilo de aprendizaje, el locus de control, las tendencias 
atribucionales etc. Podemos citar también el nivel de auto-exigencia (la búsqueda de la excelencia, el perfeccionismo en el caso más extremo), la percepción de dominio de las capacidades exigidas por la situación (sentimiento de competencia), la mayor o menor tolerancia al fracaso y otras entidades bien conocidas, como la motivación de logro.

El estilo cognitivo constituye uno de los factores que se anhelan como esenciales en la construcción del modelo, ya que, como decía antes, por encima de las características objetivas del ambiente se sitúa la apropiación que hace la persona del mismo y eso depende precisamente del estilo cognitivo. Ya Messik (1979) incluyó entre las dimensiones de los estilos de aprendizaje una amplia serie de factores que tienen una relación directa con esto. Entre ellas, podemos recordar la dependencia o independencia de campo (comprensión de elementos del medio de forma global e integrada en su contexto o de forma aislada y separada del entorno), la articulación de campo (maneras de percibir los estímulos complejos), los estilos de conceptuación (estabilidad en la utilización de estímulos), la complejidad cognitiva (que está en la base de la construcción de diferentes conductas sociales), la nivelación (que se opone a la puesta en relieve, dos extremos de una dimensión referida a la forma en que las personas establecen el balance entre los distintos estímulos presentes en un determinado momento), el factor riesgo-precaución (dimensión relacionada con factores de motivación y la evaluación de la situación), la auscultación (diferencias individuales en relación con la intensidad de la experiencia), la tolerancia respecto a experiencias no convencionales (grado de aceptación de vivencias distintas de las habituales) o el grado de automatización (habilidad individual para ejercitar acciones repetitivas o rutinas).

En la misma línea, la aplicabilidad de los principios enunciados por Knowles (1988) relacionando el aprendizaje adulto al colectivo docente sugiere, entre otras cuestiones de interés, el fomento de la autonomía profesional y la independencia de criterio, el empleo de la experiencia como fuente de aprendizaje, la acomodación del aprendizaje a los cambios sociales del rol docente y la vinculación de éste a la práctica cotidiana y a las necesidades sentidas por las profesoras y profesores. Son estos algunos de los aspectos que pueden constituirse en factores incidentes en el bienestar del profesorado.

Decía que cualquier modelo explicativo ha de incorporar los elementos personales, pero de la misma forma ha de asumir los elementos del medio laboral, entendiendo como tales tanto los que afectan específicamente al establecimiento educativo (grupos sociales, clima, cultura institucional y profesional, condiciones físicas etc), como al conjunto de la profesión 
(normativa reguladora, contraprestaciones económicas y no económicas). En este sentido, parece pertinente establecer una diferenciación entre los factores correspondientes a distintos niveles contextuales (aula, centro, comunidad, sociedad), adoptando una concepción del medio como un conjunto de entidades anidadas. Cada una de ellas está insertada en otra más amplia cuyas características le afectan y, al menos en cierta medida, la condicionan.

La concurrencia de factores personales y no personales apunta a una relación compleja entre todos ellos. El bienestar (o el malestar) no es el producto del ambiente o de la persona, sino de la interacción entre ambos. Los modelos que pretendan explicar el bienestar y/o el malestar docente han de ser, por lo tanto, modelos interaccionistas o ecológicos, es decir, que basen la explicación del constructo a través de las relaciones entre las personas y los contextos de práctica.

Pero, por encima de las relaciones existentes entre la persona y su ambiente de trabajo y de las cualidades objetivas de ambos, se sitúa la forma en que esas cualidades y la interacción es evaluada por la persona. En otros términos, se produce una mediación cognitiva entre los factores incidentes en el trabajo docente y la satisfacción que se deriva de la situación. Por ello, las teorías o modelos explicativos han de ser necesariamente mediacionales, las cuales se relacionan con algunos factores ya citados, particularmente con el estilo cognitivo, principal elemento de mediación.

El carácter multifactorial ya aludido repetidamente implica, además, complejidad. El peso de los distintos factores, su naturaleza diversa y las relaciones que se establecen entre ellos no pueden ser explicados de forma simple. Esto implica que el modelo a proponerse ha de asumir la complejidad como otra de sus cualidades esenciales.

Por la misma razón, el modelo idóneo ha de asumir la dinamicidad como cualidad básica. Si intervienen factores tan diversos, la satisfacción -el bienestar o el malestar- no es permanente, sino variable en el tiempo. Por otro lado, muchos de los factores incidentes incorporan, de alguna manera, el concepto de evolución, lo que incrementa ese dinamismo del concepto central.

Una nueva cualidad que ha de incorporar el modelo es el carácter relativo de los conceptos de bienestar y malestar. En ningún caso pueden ser considerados términos absolutos. Esa relatividad sugiere el empleo de la analogía de una dimensión bipolar en cuyos extremos se sitúan los dos conceptos antagónicos, pero entendiendo (de ahí su carácter de dimensión) que, en un momento dado, un docente puede estar situado en cualquier 
punto de esa dimensión continua. Por la misma razón, la satisfacción no es una magnitud discreta, sino continúa. No existe posibilidad de medición cuantitativa, lo que desacredita los instrumentos diagnósticos basados en una medición como si se tratara de una magnitud discreta. En puridad, si asumimos la concurrencia de procesos cognitivos mediacionales, habría que plantearse si existe la posibilidad de emplear instrumentos diagnósticos diferentes del autoinforme y las técnicas etnográficas.

Por otro lado, considerando lo anterior, los modelos explicativos del bienestar docente deben ser, de forma ineludible, multifactoriales, complejos, ecológicos, dinámicos y mediacionales, además de plantear el binomio malestar-bienestar como una dimensión continua bipolar.

\section{Promover el BIEnestar}

En la medida de lo posible, los modelos deberían incorporar, además de las cualidades ya enunciadas, las formas de transición hacia estadios de mayor bienestar.

La satisfacción constituye uno de los pilares de la calidad educativa. En ese sentido, el profesorado satisfecho se encuentra en mejores condiciones para desempeñar su trabajo que el que no lo está. Por lo tanto, se hace necesario orientar la teoría sobre bienestar docente hacia la promoción de grados adecuados de satisfacción laboral mediante intervenciones de carácter formativo.

Decía antes que la resiliencia es una ruptura terapéutica porque detiene un proceso de deterioro que lleva al malestar. Pues bien, desde ese mismo punto de vista, el concepto de compromiso supone un estado personal preventivo, anterior a la resiliencia. Si la resiliencia es un proceso terapéutico que se activa ante una situación desfavorable, el compromiso es una actitud preventiva, puesto que actúa antes de que se presente aquella relación viciosa entre clima desfavorable e insatisfacción. El compromiso es el principal componente de los procesos de cambio y, por lo tanto, actúa directamente sobre el clima laboral, provocando cambios favorables en el mismo y evitando riesgos. Formar, en el compromiso, debe constituirse en objetivo primordial de los procesos de preparación del profesorado, pero las medidas para formar en el compromiso no difieren de las que exigen una formación orientada al cambio social. En este sentido, las teorías críticas y los movimientos radicales han hecho ya aportes interesantes.

Entre esas propuestas radicales se encuentra la consigna ineludible de realizar un análisis de la realidad con vistas a reconocer situaciones de represión o coerción y superar las condiciones actuales en un proceso de 
mejora social, de emancipación o de liberación. El malestar o el bienestar docente, como hemos concluido antes, proceden de la relación con el ambiente subjetivo, que es mudable, como decía. Para ese cambio, como para el cambio emancipador propuesto por las teorías críticas, se debe dotar al profesorado de herramientas para la aprehensión (observación, comprensión, interiorización), valoración y gestión del medio (capacidad para incidir en el contexto y cambiarlo). Por lo tanto, una formación basada en los presupuestos de los paradigmas radicales o críticos parece plausible para la consecución de capacidades que incrementen el compromiso profesional, que está en la base de la satisfacción laboral y del bienestar docente.

Por otro lado, si las relaciones con los colegas constituyen un elemento incidente en el bienestar o en el malestar, se debe promover el desarrollo de habilidades interpersonales en los procesos formativos. Por la misma razón, se debe formar en estrategias de cooperación y desarrollo grupal $y$, para evitar efectos indeseables de los problemas naturales derivados de las relaciones interpersonales, también en estrategias de gestión de conflicto.

El conocimiento de la realidad, al que vengo haciendo alusión, es compatible con una de las recomendaciones que se han hecho insistentemente por ciertos sectores críticos: el abandono de los modelos prescriptivos o normativos de docentes en la formación inicial del profesorado, en beneficio de los modelos descriptivos. Como se habrá deducido, los modelos normativos (cómo debe ser un docente) se contraponen a los modelos descriptivos (cómo son, qué hacen los docentes en realidad). Los primeros provocan desconcierto cuando los docentes nóveles conocen la práctica real, al confrontar ésta con la de los modelos teóricos que la formación les proporcionó. Por el contrario, los modelos descriptivos constituyen una vía para el conocimiento de las prácticas profesionales reales, evitando contradicciones paradójicas entre teoría y práctica.

Precisamente en relación con la práctica y la formación inicial, el Practicum ha de ser reorientado hacia modelos alternativos que establezcan una relación recursiva entre teoría y práctica. Un mayor contacto con los centros docentes durante la formación inicial, un análisis crítico de las vivencias de práctica, la alternancia, que eso implica, entre la clase en la Facultad y la inmersión en la práctica docente constituyen vías de mejora de la formación inicial para evitar el llamado "choque con la realidad". Esos modelos de alternancia son compatibles con la formación basada en modelos descriptivos y también con la formación orientada a la mejora de la práctica y la promoción del compromiso profesional. En ese sentido, la formación práctica ha de ser eminentemente participativa. Los contextos de práctica 
deben ofrecer oportunidades para la puesta en valor de las habilidades personales y para el contraste crítico de las prácticas establecidas.

El malestar puede ser contagioso, en el sentido de que puede constituirse en un síndrome aprendido durante los procesos de socialización o inducción profesional, particularmente durante los periodos de prácticas en centros dentro de formación inicial, aunque también durante los primeros tiempos de ejercicio profesional, en caso de coincidir con personas que presentan conductas asociadas al síndrome. Los procesos de inducción deben contemplar ese riesgo, que ya quedaría atenuado con algunas de las medidas sugeridas.

No podemos olvidar, por otro lado, que la incidencia de los factores personales en la satisfacción docente implica la concurrencia de elementos sentimentales. Promover la educación emocional en la formación inicial y permanente se convierte, por lo tanto, en una nueva exigencia.

FROM MALAISE TO COMMITMENT: INDIVIDUAL FACTORS WHICH AFFECT THE JOB SATISFACTION OF TEACHERS

ABSTRACT: Concern about the job satisfaction of teachers has shifted from a focus on malaise to a more positive trend that focuses on wellness as a core interest. Various factors, both personal and environmental, are considered from both perspectives. This paper presents a reflection on some of the individual factors which affect the job satisfaction of teachers and proposes some suggestions for analysis. Some of the possible features required by the theoretical models aimed at explaining the job satisfaction of teachers are analyzed. Finally, some guidelines are offered for a formative intervention to promote teachers' welfare.

KEYWORDs: Teachers' malaise. Teachers' efforts. Job satisfaction.

DO MAL-ESTAR AO COMPROMISSO: OS FATORES INDIVIDUAIS NA SATISFAÇÃO LABORAL DOS DOCENTES

RESUMO: A preocupação pela satisfação laboral do professorado tem mudado desde um enfoque centrado no mal-estar até uma orientação mais positiva que focaliza o bem estar como núcleo de interesse. Em ambas perspectivas são considerados múltiplos fatores, tanto de índole pessoal como ambiental. Este artigo faz uma reflexão sobre alguns dos fatores individuais que incidem na satisfação laboral docente e propõe várias sugestões para sua análise. São analisadas, depois, algumas das características que deveriam estar presentes nos possíveis modelos teóricos orientados a explicar a satisfação laboral do professorado. Por último, ofrecem-se algumas orientações para uma intervenção formativa que promova o bem estar docente.

PalABRAS-Chave: Mal-estar docente. Compromisso docente. Satisfação laboral. 


\section{REFERENCIAS}

GONÇALVES, A. P. Dimensiones del clima laboral. Sociedad Latinoamericana para la Calidad. 1997.

JEPSON, E. y FORREST, S. Individual contributory factors in teachers stress: the role of achievement striving and occupational commitment. British Journal of Educational Psychology, 76 (1), 183-197, 2006

MORIANA, J. y HERRUZO, J. Estrés y burnout en profesores. International Journal of Clinical and Health Psychology, 4 (3), 597-621, 2004.

OBIPD. Seminario Internacional "El malestar y bienestar en la profesión docente. Políticas, prácticas y experiencias". Barcelona. Notas personales. 2012

OPECH (Observatorio Chileno de Políticas Educativas) (2008). Tensiones en la profesión docente. Documento de trabajo número 5. Consultado el 10 de mayo de 2012 en www.opech.ch

PARRA, M. La salud de los docentes: revisión de líneas emergentes en la investigación y la intervención. Santiago de Chile: UNESCO-OREALC, 2007.

REYES, M. Teorías personales del profesorado en formación sobre las organizaciones educativas. Tesis doctoral. Universidad de Huelva, 2000.

REYES, L. y CORNEJO, R. La cuestión docente en América Latina: el caso chileno. Foro Latinoamericano de Políticas Educativas, 2008.

ROBBINS, S. P. Comportamiento organizacional. México: Prentice Hall, 1999.

UNESCO. Condiciones de trabajo y salud docente. Estudios de casos en Argentina, Chile, Ecuador, México, Perú y Uruguay. Santiago de Chile: UNESCO-OREALC, 2005.

MANUEL REYES SANTANA é doutor em Psicologia. Professor da disciplina Didática e Escola, Universidade de Huelva. Diretor do Museu de Educação da mesma universidade. Professor do Programa de PósGraduação em Educação, da Universidade de Huelva. Membro do Observatório Internacional sobre profissão docente.

E-mail:mrsant@dedu.uhu.es 